\title{
Theoretical disk spectra and black-hole spin determination
}

\author{
Agata Różańska *† \\ N. Copernicus Astronomical Center, Bartycka 18, 00-716 Warsaw, Poland \\ E-mail: agata@camk.edu.pl \\ Jerzy Madej \\ Warsaw University Observatory, Al. Ujazdowskie 4, 00-478 Warsaw, Poland \\ Mateusz Gancarczyk \\ Silesian University of Technology, Akademicka 2A, 44-100 Gliwice, Poland
}

\begin{abstract}
We present angle-dependent, broad-band theoretical spectra from slim accretion disks around stellar mass black hole. In our numerical calculations, full radiative transfer of accretion disk atmospheres was solved including effects of Compton scattering, free-free and all important boundfree transitions of 10 most abundant elements. Moreover, we included the fundamental series of lines from helium-like and hydrogen-like iron. Our results show, that for high accretion rate combined with high black hole spin temperature of inner radii in the disk is of the order of $10^{7} \mathrm{~K}$. Lines of highly ionized iron can be seen in absorption. Continuum X-ray spectra exhibit numerous absorption edges of heavy elements and this shape affects determination of hardening factor, which is used to measure black hole spin. We show that the value of total disk hardening factor strongly depends on the method of its determination. Therefore, black hole spin measurements using total disk hardening factors are highly uncertain.
\end{abstract}

Fast X-ray timing and spectroscopy at extreme count rates: Science with the HTRS on the International $X$-ray Observatory - HTRS2011,

February 7-11, 2011

Champéry, Switzerland

*Speaker.

${ }^{\dagger}$ A footnote may follow. 


\section{Introduction}

From theoretical point of view, accreting matter cannot spin up black hole. Therefore, the value of black hole spin is of primordial origin, and it is vital to measure it in real sources. One of methods to estimate black hole spin is the fitting of continuum spectrum shifted by a hardening factor along the photon energy axis to the X-ray data of accreating black hole binaries.

It is widely believed, that emission originating from an accretion disk around the black hole of stellar-like mass is of multi-blackbody shape (Mitsuda et al. 1984, Dotani et al. 1997). Individual rings of the accretion disk exhibit various effective temperatures, and they contribute to the total disk spectrum integrated over radii. Multicolor disk model (MCD) still does not explain some spectral details (Merloni, Fabian, Ross 2000; Kubota et al. 2010) but up to now there is no better explanation for the observed continuum.

We construct the model of total disk spectrum, by computing radiative transfer in individual rings with careful treatment of Compton scattering on free electrons. We derive theoretical total disk hardening factor, which can be used for constraining the real black hole spin, with two different methods. We show, that due to many absorption edges on highly ionized heavy elements the value of hardening factor depends on the method of its derivation, which significantly affects the final value of black hole spin.

\section{Method of computations}

We present angle-dependent, broad-band intensity spectra from accretion disks around black hole of 10 solar masses. We attempted to reconstruct continuum and line spectra of X-ray binaries in soft state, i.e. those dominated by the disk component of multi-temperature shape. In our computations, disks are assumed to be slim (Abramowicz et al. 1988), which means that the radial advection was taken into account while computing the effective temperature of the disk.

In the first stage of calculations we solve global two-dimensional, hydrodynamical, relativistic slim disk model presented in Sagdowski et al. (2010). As the output we obtained the radial distribution of effective temperature and vertical gravity on the disk surface. Both quantities are input parameters in our radiative transfer calculations. For each global model we divided disk into $n=17$ rings centered at the distances between the innermost stable circular orbit (ISCO) up to about $r / r_{S c h w}=100$, where $r_{S c h w}=2 G M / c^{2}$. As the black hole rotates, the ISCO and event horizon moves closer to the black hole. Therefore, in case of a rotating black hole we can receive also radiation from below $r / r_{S c h w}=3$, which defines the location of ISCO in non-rotating case.

On each discrete ring we performed full radiative transfer calculations assuming that the disk atmosphere remains in hydrostatic and radiative equilibrium. We take into account effects of Compton scattering, free-free and all important bound-free transitions of 10 most abundant elements. Moreover, we include here the fundamental series of iron lines from helium-like and hydrogenlike ions. Ionization populations are computed assuming LTE equation of state for ideal gas. The method allows for a large relative photon-electron energy exchange at the time of Compton scattering. Therefore, we are able to reconstruct Compton scattering of photons with energy approaching or even exceeding the electron rest mass (Madej \& Różańska 2004). In all cases, disk matter is assumed to have solar-like chemical composition. Number abundances of elements relative to 
hydrogen, $\left(\mathrm{N}_{\text {elem }} / \mathrm{N}_{H}\right)$ equal to: 1.00 (for $\left.\mathrm{H}\right), 9.54 \times 10^{-2}(\mathrm{He}), 4.72 \times 10^{-4}(\mathrm{C}), 9.65 \times 10^{-5}$ $(\mathrm{N}), 8.55 \times 10^{-4}(\mathrm{O}), 3.84 \times 10^{-5}(\mathrm{Ne}), 4.17 \times 10^{-5}(\mathrm{Mg}), 4.94 \times 10^{-5}(\mathrm{Si}), 1.64 \times 10^{-5}(\mathrm{~S})$, $6.58 \times 10^{-5}(\mathrm{Fe})$.

Detailed equations and the way of model computation were presented in our recent paper (Różańska et al. 2011). Here we discuss only non-irradiated models in order to determine their hardening factors.

\section{Theoretical disk spectra}

We computed 3+2 models of accretion disks for the accretion rates $\dot{m}=0.01,0.1$ and 1 in units of the Eddington accretion rate. For all values of the accretion rate we consider non-rotating black hole $a=0$, while for two lowest values of $\dot{m}$ models with $a=0.98$ were also computed.

At the last step of computations we integrate energy and angle dependent spectra emitted from individual rings over disk surface according to the usual formula:

$$
I_{E}^{t o t}(\mu)=2 \pi \sum_{n=1}^{n=N} I_{E}^{(n)}(\mu) R_{n} \Delta R_{n}
$$

where $n$ is a ring number, $\mu$ is the cosine of viewing angle, and $\Delta R_{n}$ is the width of the $n$-th ring.

Our code allows us to compute the structure of disk atmospheres over very large range of electron scattering optical depths starting from $\tau_{e s}=10^{-8}$ up to $\tau_{e s}=10^{5}$. Furthermore, we were able to reproduce the continuum spectrum from deep infrared of $0.4 \mathrm{eV}$ up to hard X-rays of 400 $\mathrm{keV}$. We solve the radiative transfer problem on the grid of 175 optical depth and 2200 photon energies simultaneously.

All spectra are presented as energy dependent outgoing specific intensities, $I_{E}$, which are suitable for disk geometry. We reject presentation of monochromatic fluxes since they are relevant only to geometry of a spherical star. Our intensities are shown for 8 cosines of viewing angles, which represent angles of the Legendre quadrature. Exact values of those angles, their cosines and the type of lines used in Figs. 1, 2, 3 are: $\cos (i)=0.9801, i=11.4^{\circ}$ - solid black; $\cos (i)=0.8983$, $i=26.1^{\circ}$ - short-long dashed; $\cos (i)=0.7628, i=40.3^{\circ}$ - long dashed dotted; $\cos (i)=0.5917$, $i=53.7^{\circ}$ - short dashed dotted; $\cos (i)=0.4083, i=65.9^{\circ}$ - dotted; $\cos (i)=0.2372, i=76.3^{\circ}$ - short dashed; $\cos (i)=0.1017, i=84.2^{\circ}$ - long dashed; and $\cos (i)=0.0199, i=88.9^{\circ}$ - solid red. In further discussion we draw attention of the reader to the extreme angles: solid black line represents almost vertical direction (face-on aspect), whereas the solid red line represents almost horizontal direction (edge-on aspect).

Fig. 1 left panels present continuum spectra integrated over disk surface for all five models. Left hand part of each panel displays featureless UV, optical and near infrared spectrum of disk whereas right hand part reaches hard X-rays and iron line region. All spectra show limb-darkening over the whole range of energies. This is clearly seen in Fig. 1: intensity edge on (solid red line) is much lower than face on intensity (solid black line).

Resonance iron lines appear always in absorption for non irradiated models (Fig. 1 right panels). They are very deep, i.e central intensity in the line can drop almost three orders of magnitude (panel a) as compared to the continuum level. Our code takes into account pressure broadening of all resonance lines. And particularly Ly $\alpha$ line of He-like iron can be very wide, as seen in panels 

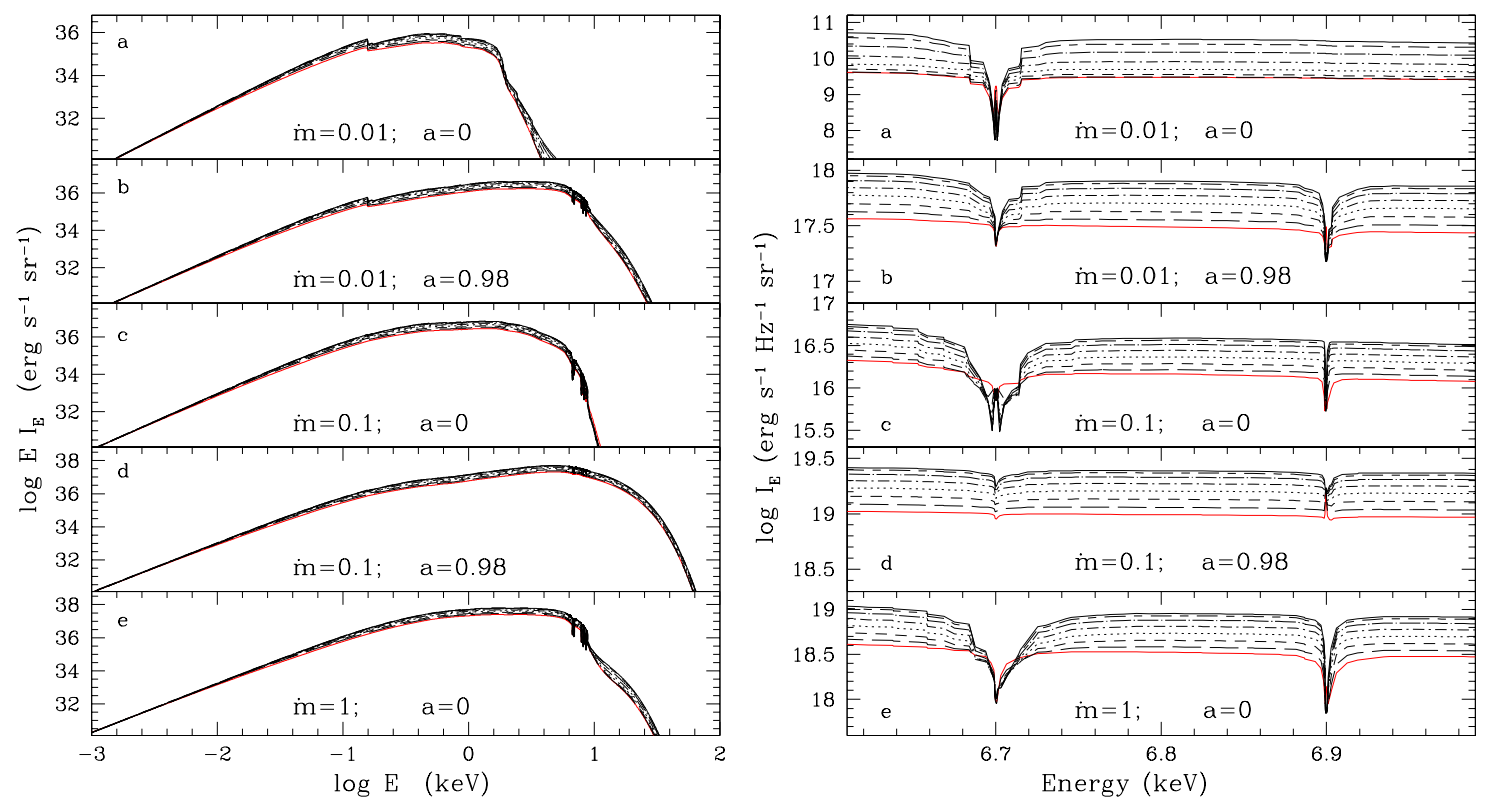

Figure 1: Broad-band intensity spectra integrated over disk surface for eight viewing angles (see Sec. 3 for the line description). Left panels represent overall continuum for all five models. The same models are enlarged on right panels in the region of iron resonant lines. No kinematic either general relativity effects were taken into account.

$\mathrm{c}$ and e. Such prominent lines certainly can be detected in present X-ray observations (Kubota et al. 2007). Accretion disks with the highest effective temperature do not show strong iron lines in absorption, and for the hottest model iron is fully ionized, as seen in panel $\mathrm{d}$.

\section{Hardening factor (color temperature correction)}

Spectrum emerging from the hot star or disk is hardened due to electron scattering in the uppermost layers of the atmosphere. In a plane-parallel atmosphere at a given $T_{\text {eff }}$, hardening factor $f$ is given:

$$
I_{E} \approx f^{-4} B_{E}\left(f \cdot T_{e f f}\right)
$$

where $B_{E}$ is the Planck function.

In the case of a hot star, when the spectrum is characterized by a single effective temperature, the easiest method of hardening factor derivation is measuring the shift of the peak flux. For neutron star spectra this method was used by Madej, Joss, Różańska (2004). Hardening factor is the ratio of energies for which scattering atmosphere and black body emission reach maximum: $f=E_{\text {max }}^{s c a t t} / E_{\max }^{B B}$. For hot, neutron star atmospheres of the order of $10^{7} \mathrm{~K}$, hardening factors are in the range: $1.3-1.84$.

Observers deal with X-ray spectra in the narrow range of photon energy measured by a satellite. So, experimentally they find hardening factor by fitting black body shape to observations in a fixed energy band. Therefore, Suleimanov, Poutanen, Werner (2011) suggested that theoretical $f$ 
should be derived minimizing the sum:

$$
\sum_{j=1}^{J}\left[F_{E}-f^{-4} B_{E}\left(f \cdot T_{e f f}\right)\right]^{2},
$$

where the theoretical flux, $F_{E}$, and the black body emission were computed for $J$ values of energy in the range: $3 \mathrm{keV}<E<20 \mathrm{keV}$. This energy range corresponds to the very often used PCA detector of the RXTE observatory. The above formula represents simple least squares fitting to the theoretical flux.

Suleimanov et al. (2011) have shown that for high luminosities, i.e. for $T_{e f f}>10^{7} \mathrm{~K}$, hardening factors computed by fitting method agree very well with maximum shift method, used by Madej et al. (2004). Nevertheless, for neutron star atmospheres with $T_{\text {eff }}<10^{7} \mathrm{~K}$, least square fitting method gives values of $f$ significantly higher than obtained by Madej et al. (2004).

\section{Total disk hardening factor}

In case of accretion disk the emission is multi-temperature, since at each given distance from the black hole we calculate new atmospheric model with different effective temperature. Therefore, at each ring we can derive a separate hardening factor, and construct radius dependent discrete function $f(R)$. On the other hand, we can integrate local intensities over the disk surface (Eq. 3.1) and compute the total disk hardening factor $f_{\text {tot }}$, following two above methods adopted to the accretion disk case. Note, that from physical point of view the total disk hardening factor does not have simple meaning of color temperature correction (energy shift).

First, we derive $f_{\text {tot }, 1}$ for all five disk models using method 1: maximum shift of the total intensity emitted by the disk atmosphere (Eq. 3.1) vs. multi-temperature black body emission i.e.

$$
B_{v}^{t o t}=2 \pi \sum_{n=1}^{n=N} B_{v}\left[T_{e f f}\left(R_{n}\right)\right] R_{n} \Delta R_{n}
$$

Resulting total hardening factors obtained by the first method for all five models are given in Table 1 in second column.

In the second method, we again fit multi-temperature black body emission corrected for the local $f(R)$ to the theoretical disk intensity in the energy range: $3 \mathrm{keV}<E<20 \mathrm{keV}$. Using the least squares fitting we minimize the sum:

$$
2 \pi \sum_{j=1}^{J}\left[\sum_{n=1}^{n=N} I_{E}^{(n)}(\mu) R_{n} \Delta R_{n}-\frac{1}{\left(f_{\text {tot }, 2}\right)^{4}} \sum_{n=1}^{n=N} B_{v}\left[f\left(R_{n}\right) \cdot T_{e f f}\left(R_{n}\right)\right] R_{n} \Delta R_{n}\right]^{2}
$$

Fig. 2 illustrates the first method for two sample disk models. One can see that the maximum atmospheric emission is modified by bound-free absorptions. Therefore, it is very difficult to find intensity peak modified by scattering. Resulting total hardening factors derived by the first method are very low.

Four examples of the total disk spectra in the range from 3-20 keV are presented in Fig. 3. Due to absorption on highly ionized iron, intensity spectra are not smooth in this energy band. For all computed models, the least squares fitting method underestimates the total hardening factor. 
Table 1: Hardening factors for all five models computed with two methods: maximum shift, method 1 $f_{\text {tot }, 1}$, and least squares fitting in the range between 3 and $20 \mathrm{keV}$, method $2-f_{\text {tot }, 2}$.

\begin{tabular}{ccc}
\hline \hline model & $f_{\text {tot }, 1}$ & $f_{\text {tot }, 2}$ \\
\hline$\dot{m}=0.01, a=0$ & 0.87 & 1.16 \\
$\dot{m}=0.01, a=0.98$ & 0.92 & 1.14 \\
$\dot{m}=0.1, a=0$ & 1.28 & 1.01 \\
$\dot{m}=0.1, a=0.98$ & 1.25 & 1.05 \\
$\dot{m}=1, a=0$ & 1.54 & 0.97 \\
\hline \hline
\end{tabular}
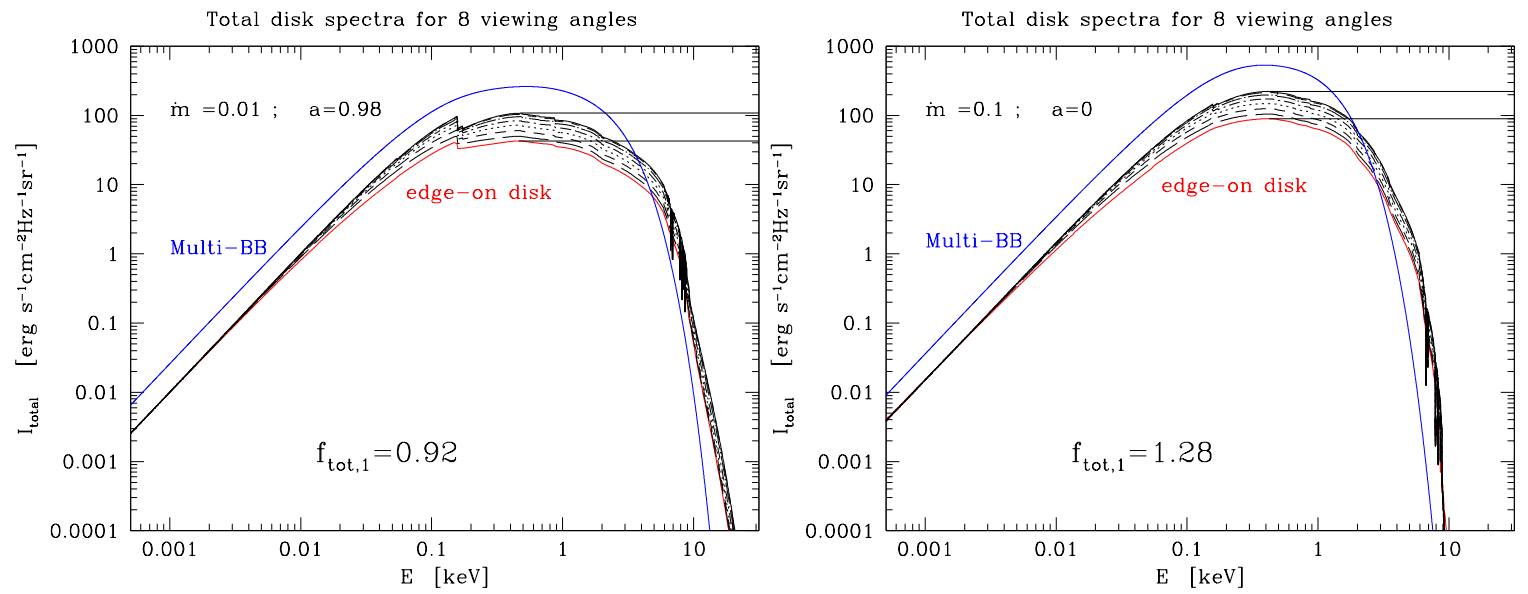

Figure 2: Two examples of $f_{t o t, 1}$ derivation, using the maximum shift method. Total disk spectra resulting from our computations are presented viewing angles listed in Sec. 3. Blue line represents multi-temperature emission integrated over disk surface. Two horizontal lines mark the position of maximum, which is strongly affected due to bound-free absorption on heavy elements

\section{Summary}

In this paper we present numerical computations of the intensity spectra of five accretion disks circulating around stellar mass black hole in the binary system. The models were computed assuming various accretion rates and two spin values of the central black hole. Effects of general relativity were not taken into account in our models.

We show, that the disk in a black hole binary is hot enough to produce strong absorption resonant lines of iron. Bound-free absorption from heavy elements affects the region of maximum emission from accretion disks, and it is hard to derive the total disk hardening factor using casual method of maximum shift, which appears to be very small with the value of 0.9-1.3.

In previous papers total disk hardening factors were computed by Shimura, Takahara (1993) in the case of only scattering atmospheres, and by Done, Davis (2008) for all relevant opacities taken into account. In the former paper disk hardening factors were rather large since absortpion was not taken into account. The latter paper presents values of hardening factor from 1.5-2.2, and those models are implemented to the XSPEC X-ray fitting package.

Our results significantly differ from those presented by previous authors. Due to strong opacities of various heavy elements between 3-20 keV, fitting method (number 2) underestimates total 

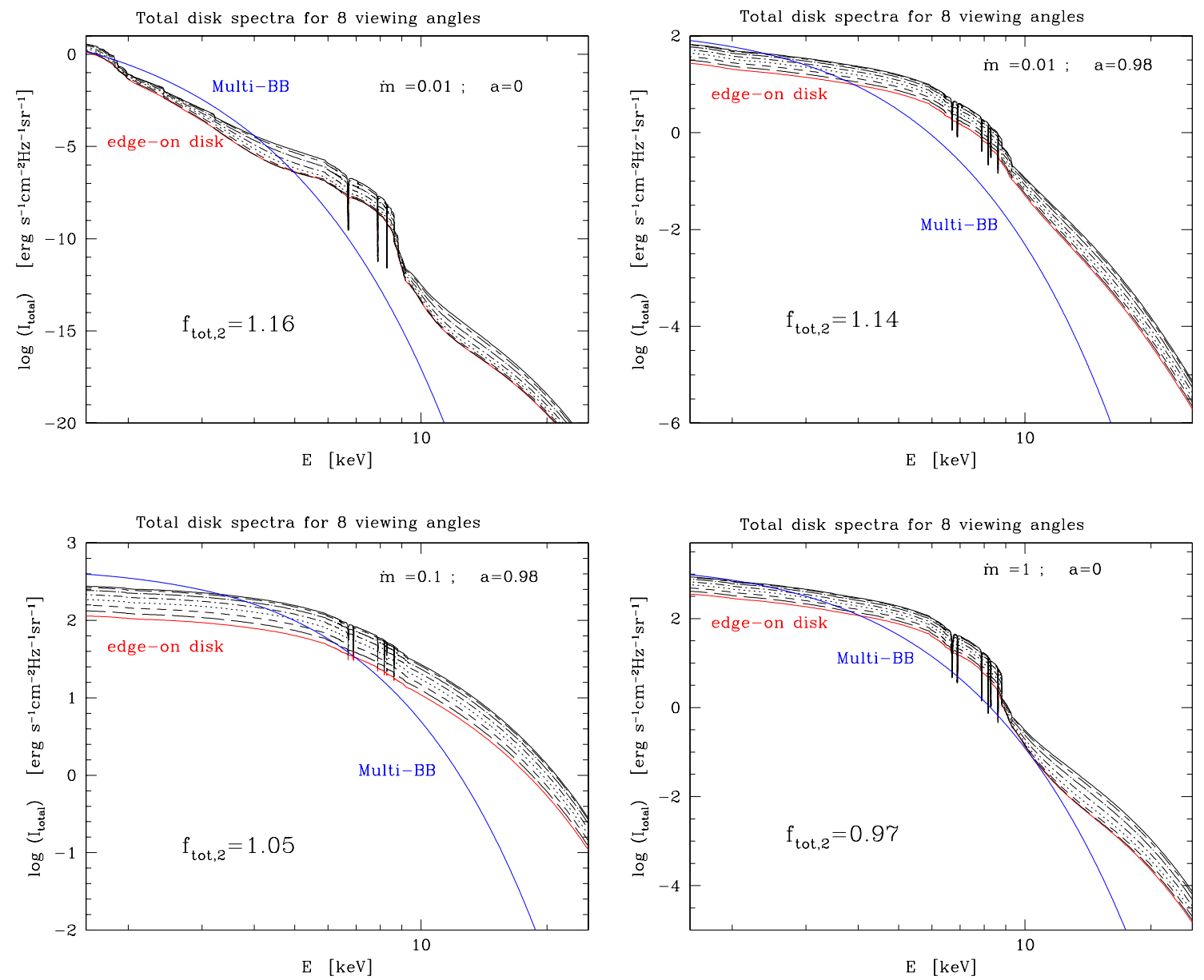

Figure 3: Four examples of $f_{t o t, 2}$ derivation, using least squares fitting method in the range between 3 and $20 \mathrm{keV}$. Multi-temperature black-body emission presented by blue line is computed using Eq. 5.1.

disk hardening factor. Therefore, black hole spin determination based only on one grid of models available in XSPEC is questionable. We anticipate re-discussion of our hardening factors in accretion disk models, when non-LTE effects in equation of state and opacities will be taken into account in the near future.

Acknowledgements: This research was supported by the Polish Ministry of Science and Higher Education grant No. N N203 511638.

\section{References}

[1] Abramowicz M. A., Czerny B., Lasota J. P., Szuszkiewicz E., 1988, ApJ, 332, 646

[2] Done C., Davis S.W., 2008, ApJ, 683, 389

[3] Dotani T., Inoue H., Mitsuda K., et al., 1997, PAJL, 485, L87

[4] Kubota A., Dotani T., Cottam J. et al., 2007, PASJ, 59, 185

[5] Kubota A., Done C., Davis S. W., 2010, ApJ, 714, 860

[6] Madej J., Joss P.C., Różańska A., 2004, MNRAS, 347, 1266 
[7] Merloni A., Fabian A. C., Ross R. R., 2000, MNRAS, 313, 193

[8] Mitsuda K., Inoue H., Koyama K., et al., 1984, PASJ, 36, 741

[9] Różańska A., Madej J., Konorski P., Sa̧dowski A., 2011, A\&A, 527, 47

[10] Shimura T., Takahara F., 1993, ApJ, 419, 78

[11] Suleimanov V., Poutanen J., Werner K., 2011, A\&A, 527, A139 